\title{
Blainvillea rhomboidea: CONSTITUINTES QUÍMICOS E ATIVIDADE CITOTÓXICA
}

\author{
Regina Ferreira Gomes, Hélcio Silva dos Santos e Maria Rose Jane R. Albuquerque* \\ Coordenação de Química, Centro de Ciências Exatas e Tecnologia, Universidade Estadual Vale do Acaraú, CP D-3, \\ 62040-340 Sobral - CE, Brasil
}

Otília Deusdênia L. Pessoa

Departamento de Química Orgânica e Inorgânica, Centro de Ciências, Universidade Federal do Ceará, CP 12200 , 60021-970 Fortaleza - CE, Brasil

Leticia V. Costa Lotufo, Claudia do Ó Pessoa, Manoel Odorico de Moraes e Felipe A. R. Rodrigues

Departamento de Fisiologia e Farmacologia, Universidade Federal do Ceará, CP 3157, 60430-270 Fortaleza - CE, Brasil

Recebido em 13/9/09; aceito em 21/1/10; publicado na web em 3/5/10

\begin{abstract}
Blainvillea rhomboidea: CHEMICAL CONSTITUENTS AND CYTOTOXIC ACTIVITY. The phytochemical investigation of the ethanol extract from the aerial parts of Blainvillea rhomboidea (Asteraceae) resulted in the isolation and characterization of 8 -tigloyloxy-grazielia acid, together with the flavonoids derrone, acacetin, luteolin and luteolin 7-methyl ether, and $p$-(1-methylethan-1-ol)-phenol. The structures of all compounds were determined by spectroscopic methods $\left({ }^{1} \mathrm{H}\right.$ and ${ }^{13} \mathrm{C}$ NMR and HREIMS) and comparison with published spectral data. The flavonoids luteolin and 7-O-metyl-luteolin, isolated from the active dichloromethane fraction, showed moderate cytotoxic activity.
\end{abstract}

Keywords: Blainvillea rhomboidea; Asteraceae; flavonoids.

\section{INTRODUÇÃO}

O gênero Blainvillea, pertencente à família Asteraceae, tribo Helianteae, subtribo Ecliptinae, é representado por apenas 10 espécies de dispersão pantropical. ${ }^{1-3}$ Destas, apenas cinco têm sido investigadas quanto a sua composição química: $B$. acmella, $B$. gayana, B. dichotoma, B. latifolia e B. rhomboidea. De acordo com estes estudos, as plantas deste gênero são produtoras em potencial de lactonas sesquiterpênicas de diferentes tipos esqueletais, tais como melampolidos, germacranolidos e guaianolidos. ${ }^{1-3}$ Classes de compostos, como diterpenos, ${ }^{4}$ derivados acetilênicos, ${ }^{5}$ triterpenos e esteroides, ${ }^{6}$ também são relatadas.

Blainvillea rhomboidea, conhecida popularmente como erva-palha ou picão grande é uma planta anual, de porte herbáceo com ramos geralmente marrom-avermelhados, medindo cerca de 80 a $160 \mathrm{~cm}$ de altura. $^{7}$ Esta espécie, como normalmente se desenvolve em grandes populações, é considerada indesejável, invasora de plantações e pastos. Inicialmente, estudou-se a composição química e a atividade antimicrobiana do óleo essencial das folhas e flores de exemplares desta espécie. Dos 18 componentes identificados nos óleos, 5-indanol (14,5\%), p-cimen-8-ol (10,1\%), $\beta$-cariofileno $(9,6 \%)$ e óxido de cariofileno $(9,6 \%)$ foram os principais constituintes. ${ }^{8} \mathrm{O}$ óleo essencial das flores, cujo principal componente foi o indanol, ausente no óleo das folhas, mostrou atividade antimicrobiana. ${ }^{8}$ Concluindo o estudo químico de B. rhomboidea, descreve-se neste trabalho o resultado obtido com a prospecção química do extrato etanólico das partes aéreas. Concomitante ao estudo fitoquímico foi investigada a atividade citotóxica dos extratos hexânico e etanólico de frações provenientes dos extratos ativos e de alguns constituintes químicos isolados.

\section{RESULTADOS E DISCUSSÃO}

O extrato etanólico da parte aérea de $B$. rhomboidea foi submetido à partição líquido-líquido utilizando os solventes hexano, $\mathrm{CH}_{2} \mathrm{Cl}_{2} \mathrm{e}$

*e-mail: rjane_7@hotmail.com
AcOEt. O fracionamento cromatográfico da fração hexano resultou no isolamento dos flavonoides 5,4'-di-hidroxi-6,6-dimetil-4,5-dihidropirano-[2,3:7,8]isoflavona (derrona) $(\mathbf{1})^{9}$ e acacetina $(2) .{ }^{10} \mathrm{Da}$ fração $\mathrm{CH}_{2} \mathrm{Cl}_{2}$ foi isolada uma lactona sesquiterpênica caracterizada como ácido $8 \beta$-tigloiloxi-grazielia (4), os flavonoides luteolina (3) e o éter 7-metil-luteolina $(6)^{11}$, além do derivado fenólico, $p$-(1metil-etan-1-ol)-fenol (5). As estruturas desses compostos foram determinadas por métodos espectroscópicos, em particular $\mathrm{RMN}{ }^{1} \mathrm{H}$ e ${ }^{13} \mathrm{C}$, uni- e bidimensionais (COSY, HSQC, HMBC e NOESY) e comparação com dados da literatura.

$\mathrm{O}$ composto $\mathbf{4}$ foi isolado como um óleo incolor. O espectro de massas de alta resolução, no modo negativo, forneceu o pico em $\mathrm{m} / \mathrm{z} 359.1495[\mathrm{M}-\mathrm{H}]^{*}$, permitindo determinar a fórmula molecular $\mathrm{C}_{20} \mathrm{H}_{24} \mathrm{O}_{6}$, indicando 9 insaturações. Seu espectro no infravermelho exibiu uma banda larga centrada em 3505 compatível com deformação axial de grupo-OH de ácido carboxílico, bandas intensas em 1762 e $1711 \mathrm{~cm}^{-1}$ compatíveis com grupos carboxilas de ácido carboxílico e éster, além de absorções na faixa de 1256 a $1038 \mathrm{~cm}^{-1}$ características de ligação C-O.

O espectro de $\mathrm{RMN}{ }^{1} \mathrm{H}$ apresentou sinais correspondentes a três grupos metilas ligados a carbono $\mathrm{sp}^{2}$, em $\delta 1,85$ (s, 3H-15), 1,75 (s, $3 \mathrm{H}^{-5}$ ') e 1,73 (d, $J=7,0 \mathrm{~Hz}, 3 \mathrm{H}-4^{\prime}$ ), os quais no espectro HSQC mostraram correlação com os sinais de carbono em $\delta 17,5 ; 14,4$ e $12,2$, respectivamente. Os sinais em $\delta 6,12$ (d, $J=3,0 \mathrm{~Hz}, \mathrm{H}-13 \mathrm{a})$ e 5,63 (d, $J=3,0 \mathrm{~Hz}, \mathrm{H}-13 \mathrm{~b}$ ), ambos correlacionados ao sinal de carbono em $\delta 120,2$, revelaram a presença de uma dupla ligação gem-dissubstituída, enquanto os sinais em $\delta 5,13(\mathrm{~d}, J=8,0 \mathrm{~Hz}$, H-5), 5,92 (dd, $J=12,8$ e 4,3 Hz, H-1) e 6,77 (q, $J=7,0$ Hz, H-3'), correlacionados aos sinais de carbono em $\delta 126,5,148,9$ e 138,3, respectivamente, foram atribuídos a duplas ligações, incluindo a de um grupo tigloíla, corroborando com a feição quarteto exibida pelo sinal em $\delta 6,77$. Os sinais em $\delta 5,82$ (d, $J=6,3 \mathrm{~Hz}, \mathrm{H}-8)$ e $5,14(\mathrm{~m}$, H-6) foram compatíveis com hidrogênios oximetínicos. O espectro de $\mathrm{RMN}{ }^{13} \mathrm{C}$-CPD apresentou sinais correspondentes a 20 átomos de carbono, cujo padrão de hidrogenação foi determinado através do espectro DEPT 135. Com base nas duas técnicas determinou-se: sete 
carbonos não hidrogenados, todos com hibridação $\mathrm{sp}^{2}$, incluindo os sinais em $\delta 169,9$ (C-12), 168,9 (C-14) e 166,9 (C-1') os quais foram atribuídos às carboxilas conjugadas de lactona, ácido carboxílico e éster, respectivamente. Três carbonos metilênicos, um dos quais em $\delta 120,2$; seis carbonos metínicos, sendo três $\mathrm{sp}^{2}$ em $\delta 148,9 ; 126,5$ e 138,3 e dois oxigenados em $\delta 76,5$ e 71,0, além de três metilas. Estes dados foram compatíveis com a estrutura de uma lactona sesquiterpênica, esterificada com um grupo tigloíla, cuja posição foi definida através da correlação do sinal de hidrogênio em $\delta$ 5,82 (H-8) com o carbono em 166,9 (C-1'), no espectro HMBC. A posição do grupo ácido carboxílico foi confirmada através da correlação observada entre o sinal em 5,92 (H-1) com o carbono em 168,9 (C-14). Todos os dados espectrais acima discutidos levaram à estrutura do ácido $8 \beta$-tigloiloxi-grazielia, previamente isolado de B. rhomboidea, cuja estrutura foi determinada utilizando-se apenas dados de $\mathrm{RMN}{ }^{1} \mathrm{H}$. Portanto, este é o primeiro trabalho onde os dados de RMN de C-13 são atribuídos para este composto (Tabela 1).

Tabela 1. Deslocamentos químicos de $\mathrm{RMN}{ }^{1} \mathrm{H}(500 \mathrm{MHz})$ e ${ }^{13} \mathrm{C}(125 \mathrm{MHz})$ de $\mathbf{4}$, em acetona- $d_{6}$

\begin{tabular}{|c|c|c|c|c|}
\hline No & $\delta_{\mathrm{C}}$ & $\delta_{\mathrm{H}}$ & ${ }^{2} J_{\mathrm{C}-\mathrm{H}}$ & ${ }^{3} J_{C-H}$ \\
\hline 1 & 148,9 & $5,92(\mathrm{dd}, J=4,3$ e 12,8$)$ & 2,37 & 3,46 \\
\hline 2 & 26,7 & $2,37(\mathrm{~m}, 2 \mathrm{H})$ & & \\
\hline 3 & 39,7 & $3,46(\mathrm{~m}, 2 \mathrm{H})$ & & 1,85 e 5,92 \\
\hline 4 & 144,6 & & 1,85 & 2,37 \\
\hline 5 & 126,5 & $5,13(\mathrm{~d}, J=8,0)$ & 5,14 & 1,85 \\
\hline 6 & 76,5 & $5,14(\mathrm{~m})$ & 3,21 & \\
\hline 7 & 53,3 & $3,21(\mathrm{~m})$ & 5,14 e 5,82 & \\
\hline 8 & 71,0 & $5,82(\mathrm{~d}, J=6,3)$ & 3,21 & \\
\hline 9 & 39,2 & $3,46(\mathrm{dd}, J=14,9$ e 6,3$) / 2,37(\mathrm{~m})$ & & \\
\hline 10 & 129,1 & & & \\
\hline 11 & 139,1 & & 6,12 & 5,82 \\
\hline 12 & 169,9 & & & 5,63 e 6,12 \\
\hline 13 & 120,2 & $6,12(\mathrm{~d}, J=3,0) / 5,63(\mathrm{~d}, J=3,0)$ & & \\
\hline 14 & 168,9 & & & 5,92 e 2,37 \\
\hline 15 & 17,5 & $1,85(\mathrm{~s})$ & & \\
\hline 1 ' & 166,9 & & & $\begin{array}{c}1,75,5,82 \mathrm{e} \\
6,77\end{array}$ \\
\hline 2 ' & 127,9 & & 1,75 & 1,73 \\
\hline 3 ' & 138,3 & $6,77(\mathrm{q}, J=7,0)$ & 1,73 & 1,75 \\
\hline $4^{\prime}$ & 12,2 & $1,73(\mathrm{~d}, J=7,0)$ & & \\
\hline$\underline{5}$ & 14,4 & $1,75(\mathrm{~s})$ & & 6,77 \\
\hline
\end{tabular}

Deslocamentos químicos $(\delta)$ em ppm. Multiplicidades e constantes de acoplamento $(J)$, em Hz, entre parênteses.

Paralelo ao estudo fitoquímico, investigou-se o potencial citotóxico das frações resultantes do fracionamento cromatográfico do extrato EtOH (Tabela 2), assim como dos compostos isolados.

O resultado da avaliação citotóxica mostrou que na concentração de $50 \mu \mathrm{g} / \mathrm{mL}$ a fração diclorometano apresentou inibição do crescimento celular nas duas linhagens celulares testadas - HCT-8 e MDAMB-435 - correspondentes a 58,75 e 46,65\%, respectivamente (Tabela 2). Todas as frações, com exceção da fração $\mathrm{MeOH}$, obtidas com o fracionamento cromatográfico da fração diclorometano ativa apresentaram um forte percentual de inibição sobre as duas linhagens celulares testadas (Tabela 2). A fração $\mathrm{MeOH}$, por sua vez, mostrou inibição somente na linhagem de melanoma (MDA-MB 435). Todos os compostos obtidos das frações ativas foram testados, mas somente os flavonoides luteolina (3) e o éter 7-metil-luteolina (6) apresentaram citotoxicidade. A luteolina apresentou valores de $\mathrm{IC}_{50}$ que variaram de $3,37 \mu \mathrm{g} / \mathrm{mL}$ em HCT-8 a 18,10 $\mu \mathrm{g} / \mathrm{mL}$ em MDA-MB-435, enquanto
Tabela 2. Determinação do percentual de inibição do crescimento celular em linhagens celulares de tumores humanos da fração diclorometano FDFBR* e frações resultantes do fracionamento cromatográfico da mesma

\begin{tabular}{lllll}
\hline Frações testadas & HCT-8 & DP & MDAMB-435 & DP \\
\hline FDFBR & 58,75 & 1,60 & 46,65 & 3,59 \\
Fração Hex/AcOEt 20\% & 100 & 0,16 & 97,82 & 1,21 \\
Fração Hex/AcOEt 50\% & 100 & 0,00 & 99,80 & 0,47 \\
Fração Hex/AcOEt 75\% & 100 & 0,57 & 98,22 & 1,03 \\
Fração AcOEt 100\% & 100 & 0,16 & 97,75 & 1,68 \\
Fração MeOH 100\% & 13,29 & 1,97 & 92,40 & 1,40 \\
Doxorubicina & 94,58 & 1,66 & 98,76 & 1,54 \\
\hline
\end{tabular}

*FDFBR = fração diclorometano obtida da partição do extrato etanólico de B. rhomboidea

que éter 7-metil-luteolina exibiu valores $\mathrm{IC}_{50}$ variando de $2,71 \mu \mathrm{g} / \mathrm{mL}$ em HCT-8 a 19,06 em SF-295 (Tabela 3). De fato, as propriedades anticâncer da luteolina já são amplamente discutidas na literatura, e seus efeitos citotóxicos já foram descritos para várias linhagens de células tumorais, estando relacionados ao bloqueio do ciclo celular em G2M seguido de apoptose, além de modular a atividade de várias proteínas celulares. ${ }^{12,13}$ Além disso, a luteolina mostrou-se capaz de inibir a angiogênese, previnir a carcinogênese e sensibilizar as células tumorais a agentes quimoterápicos. ${ }^{14,15}$

Tabela 3. Determinação da concentração inibitória média $\left(\mathrm{CI}_{50} \mathrm{em} \mu \mathrm{g} / \mathrm{mL}\right)$ dos compostos isolados de B. rhomboidea em linhagens celulares de tumores humanos ${ }^{\mathrm{a}}$

\begin{tabular}{lllll}
\hline Composto & HL-60 & HCT-8 & MDAMB-435 & SF-295 \\
\hline $\mathbf{1}$ & 27,47 & $>50$ & $>50$ & $>50$ \\
$\mathbf{2}$ & $>50$ & $>50$ & $>50$ & $>50$ \\
$\mathbf{3}$ & 5,17 & 3,37 & 18,10 & 12,44 \\
& $(4,076 \mathrm{a}$ & $(2,327 \mathrm{a}$ & $(13,22 \mathrm{a}$ & $(7,783 \mathrm{a}$ \\
& $6,564)$ & $4,882)$ & $24,77)$ & $19,89)$ \\
$\mathbf{4}$ & $>50$ & $>50$ & $>50$ & $>50$ \\
$\mathbf{6}$ & 5,067 & 2,713 & 13,48 & 19,06 \\
& $(4,025 \mathrm{a}$ & $(2,023 \mathrm{a}$ & $(10,85 \mathrm{a}$ & $(12,14 \mathrm{a}$ \\
& $6,379)$ & $3,639)$ & $16,73)$ & $29,93)$ \\
Doxorubicina & 0,02 & 0,04 & 0,47 & 0,25 \\
& $(0,01-0,02)$ & $(0,03-0,05)$ & $(0,35-0,65)$ & $(0,17-0,36)$ \\
\hline
\end{tabular}

${ }^{\mathrm{a} O s}$ dados apresentados em valores de $\mathrm{IC}_{50}(\mu \mathrm{g} / \mathrm{mL})$ foram calculados a partir de regressão não linear utilizando o programa Prisma versão 3.0 (GraphPad Software). Os intervalos de confiança (IC 95\%), obtidos em três experimentos independentes, com os desvios padrões são mostrados entre parênteses.

\section{PARTE EXPERIMENTAL}

\section{Instrumentação e material cromatográfico}

Os pontos de fusão foram determinados em equipamento de microdeterminação digital da Mettler Toledo com placa aquecedora FP82HT e uma central de processamento FP90. As determinações foram realizadas a uma velocidade de aquecimento de $2{ }^{\circ} \mathrm{C} / \mathrm{min}$ e não foram corrigidas. Os espectros de absorção na região de IV foram registrados em espectrômetro Perkin-Elmer, modelo 1000 com transformada de Fourier, utilizando pastilhas de $\mathrm{KBr}$ para amostras sólidas e filmes para substâncias oleosas. Os espectros de $\mathrm{RMN}{ }^{1} \mathrm{He}^{13} \mathrm{C}$, unie bidimensionais, foram obtidos em espectrômetro Bruker, modelo DRX-300 (300 MHz para ${ }^{1} \mathrm{H}$ e $75 \mathrm{MHz}$ para ${ }^{13} \mathrm{C}$ ) e Avance DRX-500 $\left(500 \mathrm{MHz}\right.$ para ${ }^{1} \mathrm{H}$ e $125 \mathrm{MHz}$ para ${ }^{13} \mathrm{C}$ ). O espectro de massa de alta resolução foi obtido em um LCMS-IT-TOF equipado com fonte de ionização por electronspray da Shimadzu. Nas cromatografias de adsorção utilizou-se gel de sílica 60 da Vetec ( $\varnothing$ um 70-230 mesh, para cromatografias gravitacionais) e Merck ( $\varnothing$ m 230-400 mesh, 
para cromatografias sobre pressão), enquanto nos fracionamentos cromatográficos por exclusão molecular foi empregado Sephadex LH20. As cromatografias em camada delgada analítica (CCDA) foram realizadas com gel de sílica 60, (Ø $\mu \mathrm{m}$ 5-40, Merck) com indicador de fluorescência na faixa de $254 \mathrm{\eta m}\left(\mathrm{F}_{254}\right)$. As substâncias foram reveladas com solução de vanilina/ácido perclórico/EtOH, seguida de aquecimento em estufa $\left(\sim 100^{\circ} \mathrm{C}\right)$, por aproximadamente 5 min.

\section{Material vegetal}

Blainvillea rhomboidea foi coletada no período de floração, no Campus da Universidade Federal do Ceará, em junho de 2005. A identificação botânica foi realizada pelo Prof. E. P. Nunes, Departamento de Biologia da UFC. A exsicata da planta, correspondente a coleta, encontra-se depositada no Herbário Prisco Bezerra (EAC) do Departamento de Biologia - UFC, sob o número de registro 33.879.

\section{Extração e isolamento}

A parte aérea $(1,5 \mathrm{~kg})$ de $B$. rhomboidea foi seca à temperatura ambiente, triturada e, em seguida, submetida à extração $(3 \times 8 \mathrm{~L})$ com etanol a frio. As soluções obtidas foram destiladas sob pressão reduzida, resultando no extrato etanólico $(85,9 \mathrm{~g})$ o qual foi dissolvido em $150 \mathrm{~mL}$ da mistura $\mathrm{MeOH} / \mathrm{H}_{2} \mathrm{O}(7: 3)$ e submetido à partição com os solventes hexano, $\mathrm{CH}_{2} \mathrm{Cl}_{2}$ e AcOEt (5 vezes $100 \mathrm{~mL}$ de cada solvente). As frações obtidas foram secas com sulfato de sódio anidro $\left(\mathrm{Na}_{2} \mathrm{SO}_{4}\right.$ ), filtradas e concentradas sob pressão reduzida, fornecendo as frações hexanica (3,9 g), $\mathrm{CH}_{2} \mathrm{Cl}_{2}(29,0 \mathrm{~g})$ e AcOEt $(1,9 \mathrm{~g})$, além do resíduo $(6,9 \mathrm{~g})$. A fração hexano $(3,9 \mathrm{~g})$ foi submetida à cromatografia sobre $40 \mathrm{~g}$ de gel de sílica utilizando os solventes hexano e AcOEt, puros ou em misturas binárias, seguido por $\mathrm{MeOH}$. Deste fracionamento foram obtidas as frações hexano/AcOEt 9,5:0,5 (1,4 g), 9:1 (0,48 g), 8:2 (0,40 g), 7:3 (0,69 g), 6:4 (0,43 g), 3,5:6,5 (0,30 g), AcOEt $(0,03 \mathrm{~g})$ e MeOH (0,093 g). A fração hexano/AcOEt 8:2 $(0,40 \mathrm{~g})$ foi submetida a uma cromatografia sobre pressão e eluída com a mistura binária de hexano/AcOEt 9:1 fornecendo 125 frações de $8 \mathrm{~mL}$. Após monitoramento em CCD, estas frações foram reunidas em 5 grupos: (A-I a A-V). O grupo A-IV (Frações 73-121, 72,0 mg) após cromatografia em gel de sílica $(5,0 \mathrm{~g})$ utilizando misturas de hexano e AcOEt em gradiente crescente de polaridade resultou no isolamento de 1 (6,0 mg; p. f. $220-223^{\circ} \mathrm{C}$; lit. $\left.216-218^{\circ} \mathrm{C}\right)^{9}$. A fração hexano/AcOEt 7:3 $(0,69 \mathrm{~g})$ foi sujeita à cromatografia em gel de sílica $(2,0 \mathrm{~g})$ e eluída com a mistura binária hexano/ $\mathrm{CHCl}_{3}$ em gradiente de polaridade, seguido de AcOEt. Foram obtidas 69 frações de $10 \mathrm{~mL}$ que, após serem analisadas por $\mathrm{CCD}$, foram reunidas em 6 grupos (B-I a B-VI). O grupo IV (Frações 35-46, 61,0 mg) após cromatografia por exclusão molecular, em Sephadex LH-20 empregando acetona/ $\mathrm{MeOH}$ 1:1 como fase móvel, culminou no isolamento de 2 (11,0 mg ; p. f. $256-257^{\circ} \mathrm{C}$; lit. $\left.254-256{ }^{\circ} \mathrm{C}\right)^{10}$. A fração $\mathrm{CH}_{2} \mathrm{Cl}_{2}(29,0 \mathrm{~g})$, oriunda do fracionamento cromatográfico inicial, foi fracionada sobre 47,0 $\mathrm{g}$ de gel de sílica utilizando os solventes hexano e AcOEt puros ou em misturas binárias, seguido de $\mathrm{MeOH}$. Ao final foram obtidas as seguintes frações: hexano/AcOEt 9:1 (0,02 g); 8:2 (0,33 g); 7:3 (0,96 g), 1:1 (2,0 g), 3,5:6,5 (6,8 g); AcOEt (9,1 g) e MeOH (9,2 g). A fração hexano/AcOEt 1:1 (2,0 g) foi submetida à cromatografia em gel de sílica (41,0 g) e eluída com os solventes $\mathrm{CHCl}_{3}$ AcOEt e $\mathrm{MeOH}$, puros ou em gradiente de polaridade, resultando em 9 frações de 100 $\mathrm{mL}$, as quais após concentradas sob pressão reduzida e analisadas por CCD foram reunidas em 7 grupos (C-I a C-VII). A fração C-IV (frações $\mathrm{CHCl}_{3}$-AcOEt 1:1,370,0 mg) foi submetida a fracionamento cromatográfico sobre Sephadex LH-20 e eluída com acetona/MeOH 1:1, resultando no isolamento de 3 (15,0 mg; p.f. 322-324; lit. > 320 $\left.{ }^{\circ} \mathrm{C}\right)^{11}$. A fração hexano/AcOEt 3,5:6,5 (6,8 g) foi sujeita à croma- tografia sobre gel de sílica $(44,0 \mathrm{~g})$ utilizando um sistema binário constituído de $\mathrm{CHCl}_{3} / \mathrm{AcOEt}$, aumentando-se a concentração de 20 em 20\%, seguido de AcOEt e MeOH. As frações obtidas após concentração do solvente resultaram nas frações $\mathrm{CHCl}_{3}(3,7 \mathrm{~g}), \mathrm{CHCl}_{3}$ / AcOEt 8:2 (1,0 g), 6:4 (650,0 mg), 4:6 (642,0 mg), 2:8 (410,0 mg), AcOEt (191,0 mg) e MeOH (320,0 mg). A fração $\mathrm{CHCl}_{3}(3,7 \mathrm{~g})$ foi submetida à cromatografia sobre 42,3 g de gel de sílica, empregando os solventes hexano e AcOEt, puros ou em misturas binárias seguido de $\mathrm{MeOH}$. Foram coletadas 27 frações as quais após análise em CCD foram reunidas em 11 grupos (D-I a D-XI). Os grupos D-III (frações 8-9, 151,9 mg) e D-VI (frações 10-11, 465,1 mg), após comparação em CCD, mostrou conter as substâncias 1 e 2, respectivamente. A fração D-V (fração 12, 691,0 mg) foi submetida à cromatografia sobre gel de sílica (7,9 g) utilizando os solventes hexano, AcOEt e $\mathrm{MeOH}$, em gradiente de polaridade. Desta coluna obtiveram-se 110 frações, as quais após análise por CCD foram reunidas em 10 grupos (E-I a E-10). O grupo E-II (frações 24-30, 209,0 mg) após cromatografias por exclusão molecular sobre Sephadex LH-20, empregando o sistema de solvente acetona/MeOH 1:1, culminou no isolamento de $\mathbf{4}(57,4$ mg). A fração $\mathrm{CHCl}_{3} / \mathrm{AcOEt}$ 8:2 (1,0 g), foi acondicionada sobre $16,5 \mathrm{~g}$ de gel de sílica e submetida a fracionamento cromatográfico, utilizando um gradiente constituído de hexano/AcOEt (8:2 a 2:8) seguido de AcOEt, resultando em 82 frações de $8 \mathrm{~mL}$. A análise por CCD permitiu reuni-las em 7 grupos (F-I a F-VII). O grupo F-III (frações 36-52, 65,0 mg) após fracionamento cromatográfico sobre Sephadex LH-20 utilizando o sistema de solvente acetona/MeOH 1:1, forneceu a substância $\mathbf{5}$ (7,4 mg). O grupo F-V (430,0 mg) após ser submetido a procedimento cromatográfico semelhante ao anterior resultou no isolamento da substância 6 (11,0 mg) (Figura 1).<smiles>COc1ccc(-c2cc(=O)c3c(O)cc(O)cc3o2)cc1</smiles>

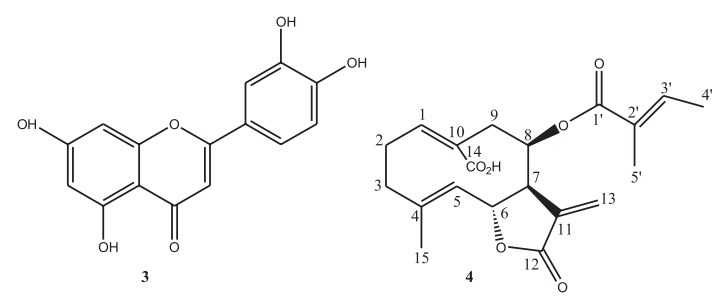<smiles>COc1ccc(C(C)(C)O)cc1</smiles><smiles>COc1cc(O)c2c(=O)cc(-c3ccc(O)c(O)c3)oc2c1</smiles>

Figura 1. Estruturas dos constituintes químicos isolados das partes aéreas de B. rhomboidea

\section{Avaliação da atividade citotóxica}

A citotoxicidade dos compostos foi avaliada em 4 linhagens tumorais, usando a análise de redução do 3-(4,5-dimetil-2-tiazolil)2,5-difenil-2H-brometo tetrazolina (MTT) ${ }^{16}$ (Sigma Aldrich Co. - St. Louis, USA). Para o experimento, as células foram distribuídas em placas de 96 poços $\left(10^{5}\right.$ células/poço para células aderentes ou $3 \mathrm{x}$ 
$10^{5}$ células/poço para células em suspensão em $100 \mu \mathrm{L}$ de meio). As frações foram testadas em concentração única de $50 \mu \mathrm{g} / \mathrm{mL}$ para determinação do percentual de inibição do crescimento celular, enquanto os compostos puros foram diluídos em concentrações que variaram de 0.09 a $50 \mu \mathrm{g} / \mathrm{mL}$ em DMSO $1 \%$. Cada composto foi adicionado em cada poço usando-se HTS (high-throughput screening, biomek 3000, Beckman Coulter, Inc. Fullerton, California, USA) e posteriormente incubado por $72 \mathrm{~h}$. O grupo controle negativo recebeu a mesma quantidade de DMSO. Depois de $69 \mathrm{~h}$ de incubação, o sobrenadante foi aspirado e acrescentado à solução do MTT $(0,5 \mathrm{mg} /$ $\mathrm{mL}$ ). Três horas depois, o produto formado do MTT, o formazan, foi dissolvido em $150 \mu \mathrm{L}$ de DMSO e a absorbância foi lida a $595 \mathrm{~nm}$ (DTX 880 Multimode Detector, Beckman Coulter, Inc. Fullerton, California, USA). Como controle positivo usou-se a doxorrubicina $(0,01$ a $5 \mu \mathrm{g} / \mathrm{mL})$ obtida da Sigma Aldrich Co. - St. Louis, MO/USA.

\section{CONCLUSÃO}

A prospecção química de $B$. rhomboidea culminou no isolamento de quatro flavonoides e de uma lactona sesquiterpênica. Embora flavonoides sejam bastante comuns em plantas superiores, inclusive na família Asteraceae, este é o primeiro relato sobre o isolamento deste tipo de composto em uma planta do genêro Blainvillea, por outro lado, as lactonas sesquiterpênicas são os constituintes mais comuns no gênero, inclusive em plantas de origem brasileira.

\section{MATERIAL SUPLEMENTAR}

Os espectros e RMN e EM estão disponíveis em http://quimicanova.sbq.org.br, em arquivo .PDF, com acesso livre.

\section{AGRADECIMENTOS}

À FUNCAP e PRONEX pelo apoio financeiro, ao CNPq pelas bolsas de estudo e de pesquisa, ao Centro Nordestino de Aplicação e Uso da Ressonância Magnética Nuclear (CENAUREMN) e Laboratório de Espectrometria de Massa do Nordeste (LEMANOR) pela obtenção dos espectros.

\section{REFERÊNCIAS}

1. Spring, O.; Zipper, R.; Vogler, B.; Lopes, J. L. C.; Vichnewski, W.; Dias, D. A.; Cunha, W. R.; Phytochemistry 1999, 52, 79.

2. Singh, P.; Sharma, A. K.; Joshi, K. C.; Jakupovic, J.; Bohlmann, F.; Phytochemistry 1985, 24, 2023.

3. Bohlmann, F.; Ziesche, J.; King, R. M.; Robinson, H.; Phytochemistry 1981, 20, 263

4. Singh, P.; Bhala, M.; Jain, R.; Jakupovic, J.; Phytochemistry 1988, 27, 609.

5. Krishnar, V.; Gupta, G. S.; Singh, P.; J. Indian Chem. Soc. 1999, 76, 689.

6. Adityachaudhury, N.; Chowdhury, A.; Phytochemistry 1972, 11, 3544.

7. Lorenzi, H.; Matos, F. J. A.; Plantas Medicinais no Brasil: Nativas e Exóticas, Plantarum: São Paulo, 2002.

8. Pires, A. M. L.; Albuquerque, M. R. J. R.; Nunes, E. P.; Melo, V. M. M.; Silveira, E. R.; Pessoa, O. D. L.; Nat. Prod. Commun. 2006, 1, 391.

9. Chibber, S. S.; Sharma, R. P.; Phytochemistry 1980, 19, 1857.

10. Costa, F. J.; Bandeira, P. N.; Albuquerque, M. R. J. R.; Pessoa, O. D. L.; Silveira, E. R.; Braz-Filho, R.; Quim. Nova 2008, 31, 1691.

11. Agrawal, P. K.; Carbon-13 NMR of Flavonoids, Elsevier: Amsterdan, 1989.

12. Zhang, Q.; Zhao, X. H.; Wang, Z. J.; Food Chem. Toxicol. 2008, 46, 2042.

13. Zhang, Q.; Zhao, X. H.; Wang, Z. J.; Toxicol. in Vitro 2009, 23, 797.

14. López-Lázaro, M.; Mini Rev. Med. Chem. 2009, 9, 31.

15. Lin, Y.; Shi, R.; Wang, X.; Shen, H. M.; Curr. Cancer Drug Targets 2008, 8, 634 .

16. Mosmann T.; J. Immunol. Methods 1983, 65, 55. 


\section{Blainvillea rhomboidea: CONSTITUINTES QUÍMICOS E ATIVIDADE CITOTÓXICA}

Regina Ferreira Gomes, Hélcio Silva dos Santos e Maria Rose Jane R. Albuquerque*

Coordenação de Química, Centro de Ciências Exatas e Tecnologia, Universidade Estadual Vale do Acaraú, CP D-3, 62040-340

Sobral - CE, Brasil

Otília Deusdênia L. Pessoa

Departamento de Química Orgânica e Inorgânica, Centro de Ciências, Universidade Federal do Ceará, CP 12200, 60021-970 Fortaleza - CE, Brasil

Leticia V. Costa Lotufo, Claudia do Ó Pessoa, Manoel Odorico de Moraes e Felipe A. R. Rodrigues

Departamento de Fisiologia e Farmacologia, Universidade Federal do Ceará, CP 3157, 60430-270 Fortaleza - CE, Brasil

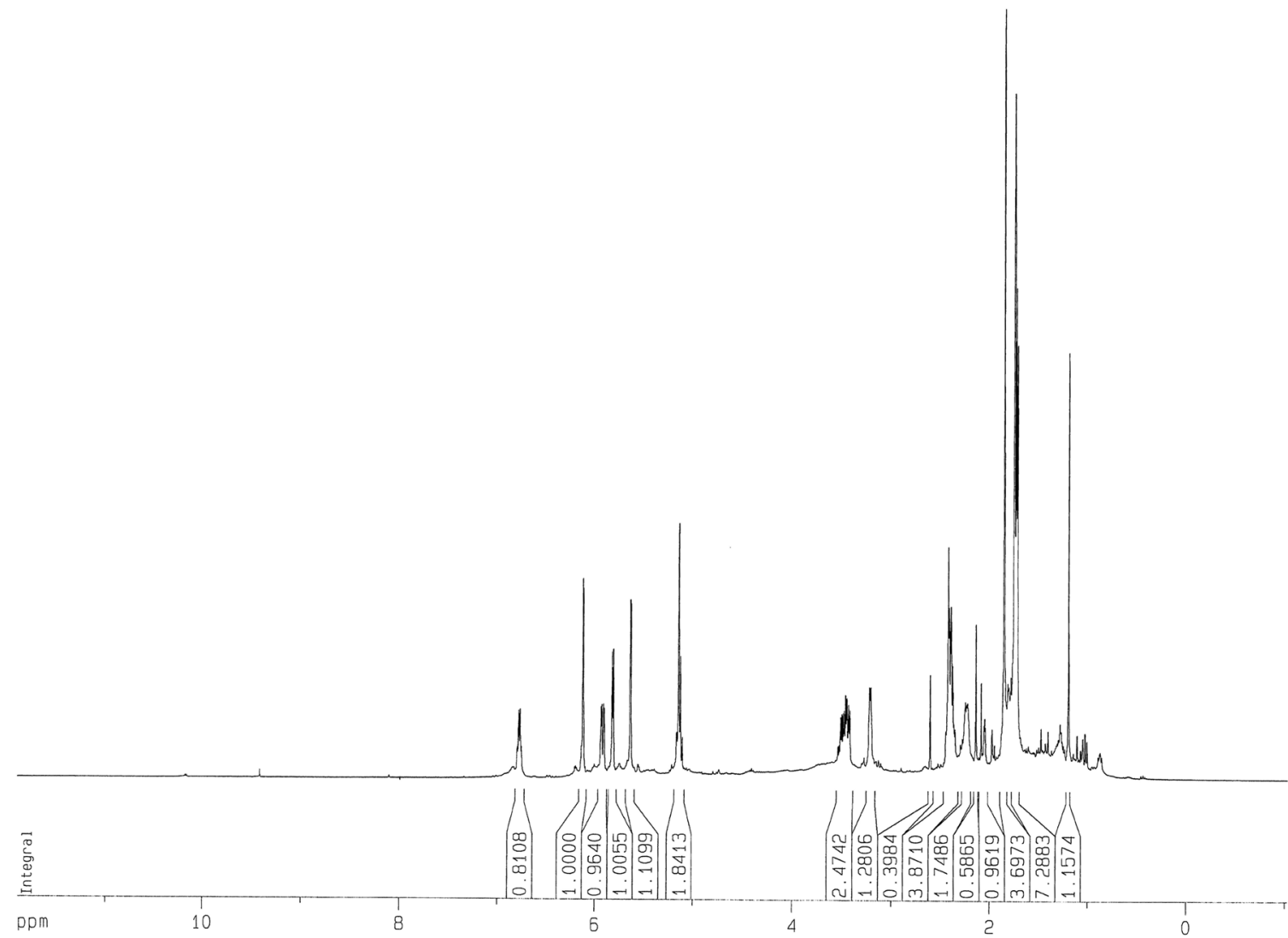

Figura 1S. Espectro de $\left.\mathrm{RMN}^{1} \mathrm{H}\left[\left(\mathrm{CD}_{3}\right)_{2} \mathrm{CO}, 500 \mathrm{MHz}\right)\right]$ do composto 4 
S2

Gomes et al.

Quim. Nova
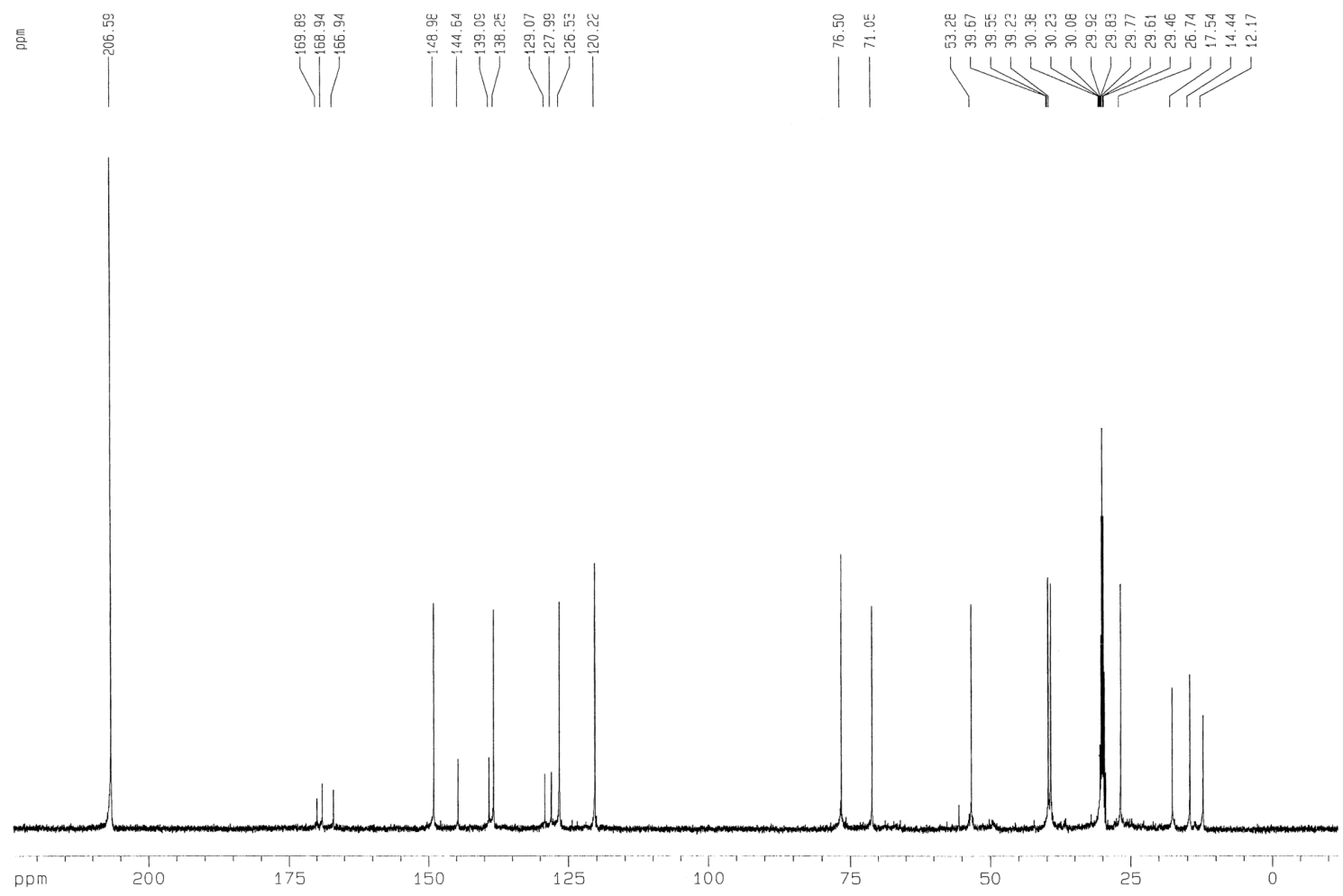

Figura 2S. Espectro de $\left.\mathrm{RMN}^{13} \mathrm{C}\left[\left(\mathrm{CD}_{3}\right)_{2} \mathrm{CO}, 125 \mathrm{MHz}\right)\right]$ do composto 4
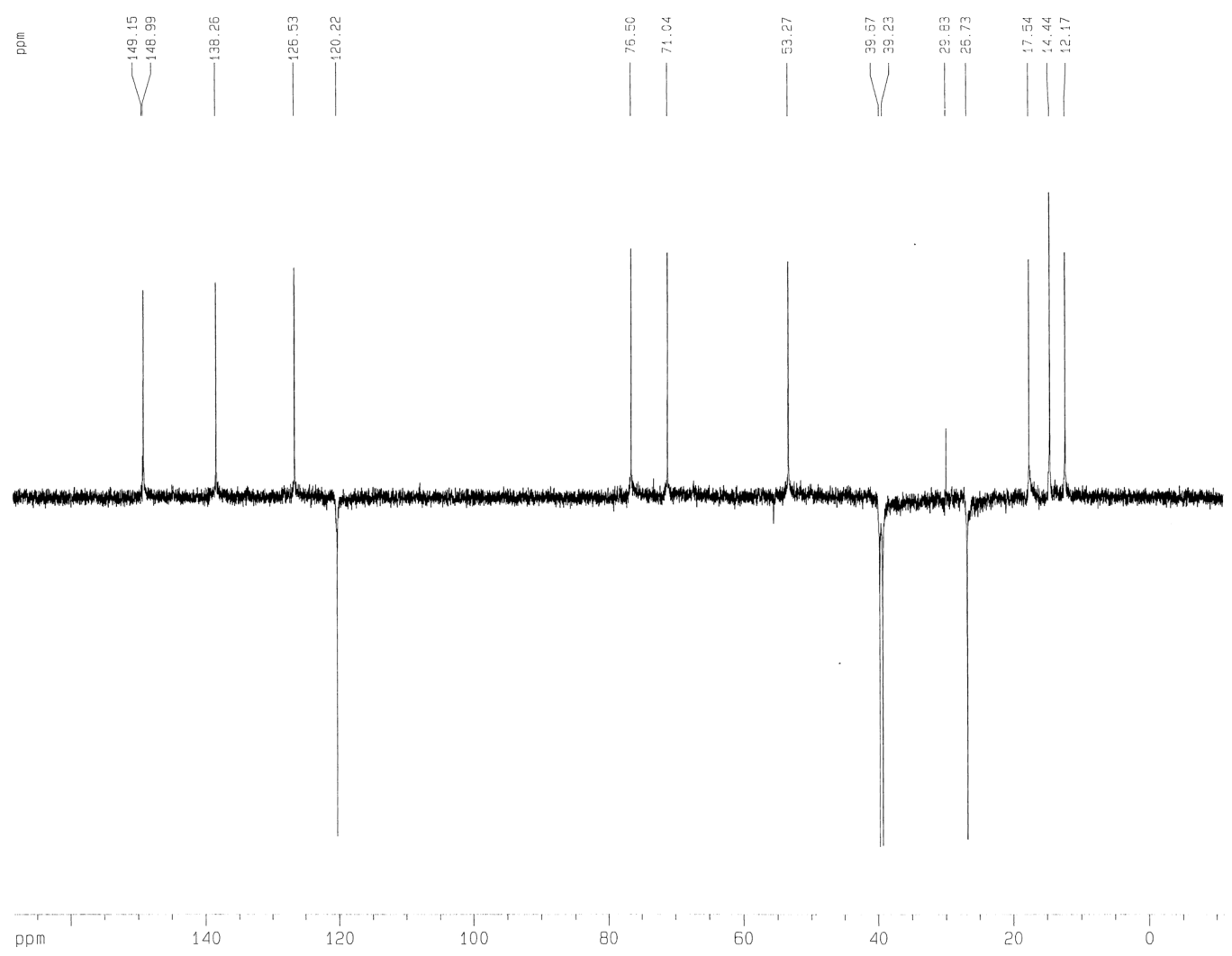

Figura 3S. Espectro de RMN DEPT-135 [( $\left.\left.\left.\mathrm{CD}_{3}\right)_{2} \mathrm{CO}, 125 \mathrm{MHz}\right)\right]$ do composto 4 


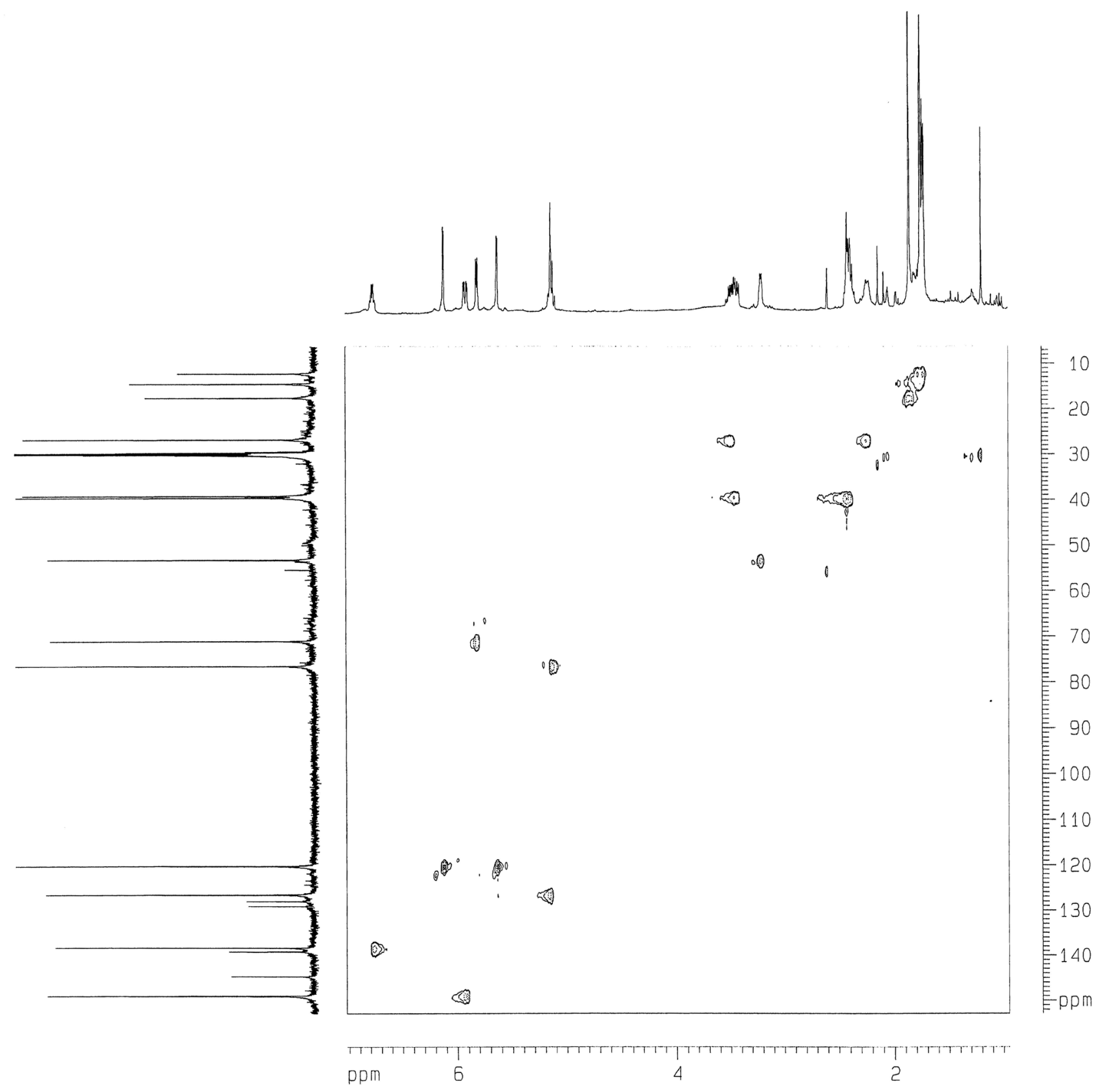

Figura 4S. Espectro de RMN HSQC $\left[\left(\mathrm{CD}_{3}\right)_{2} \mathrm{CO}\right]$ do composto 4 


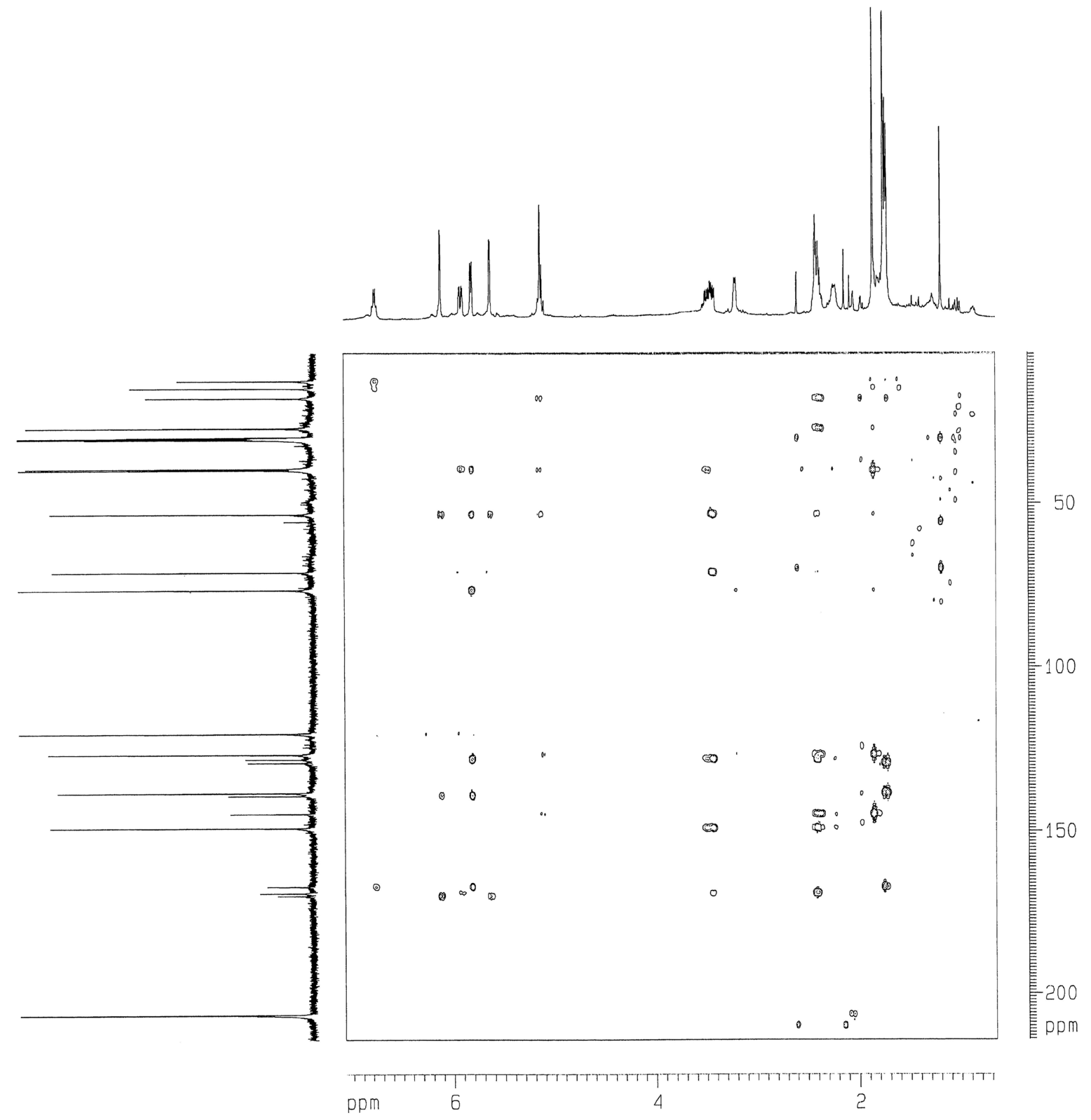

Figura 5S. Espectro de RMN HMBC $\left[\left(\mathrm{CD}_{3}\right)_{2} \mathrm{CO}\right]$ do composto 4 


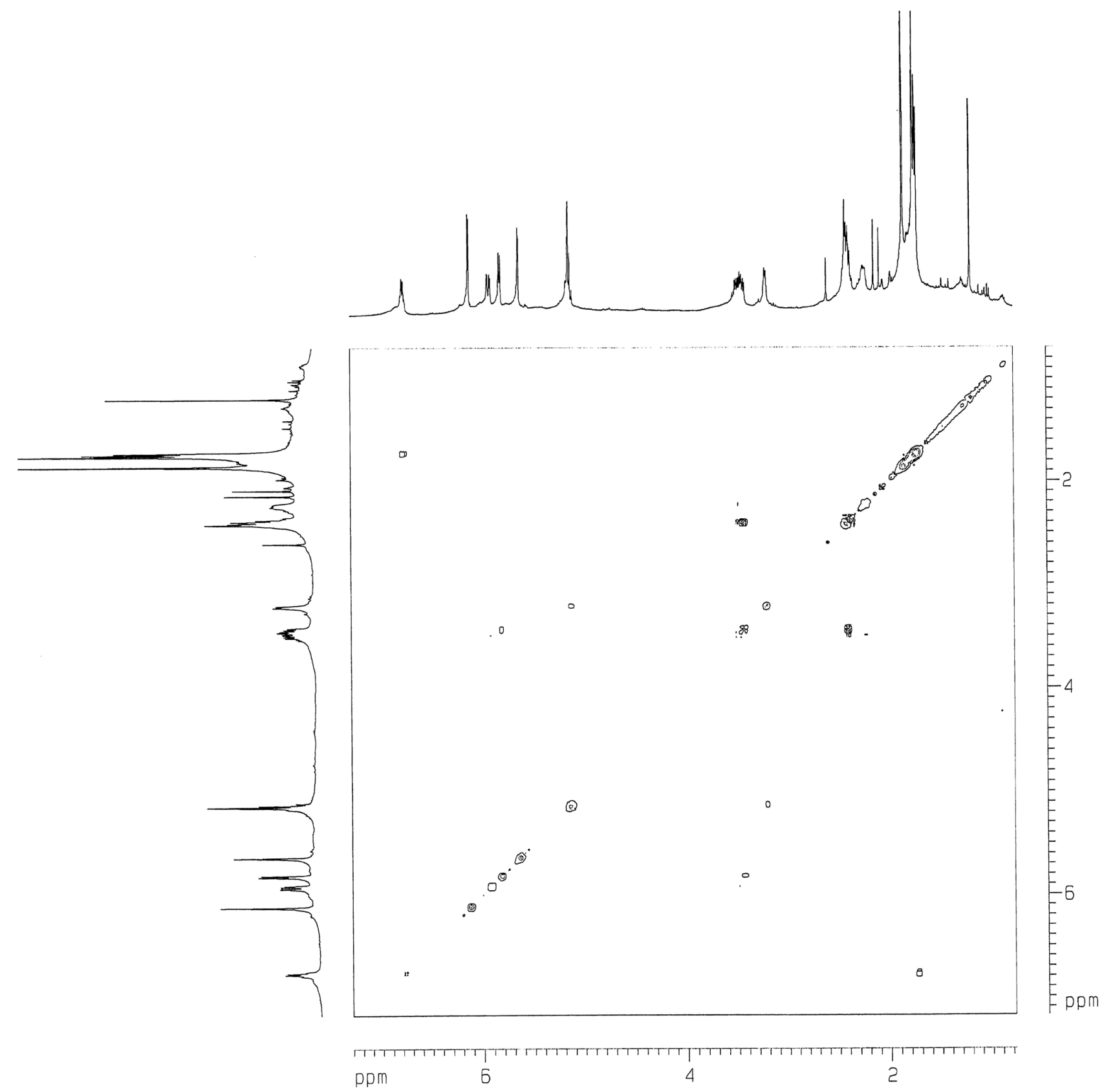

Figura 6S. Espectro de RMN COSY $\left.\left(\mathrm{CD}_{3}\right)_{2} \mathrm{CO}\right)$ do composto 4 


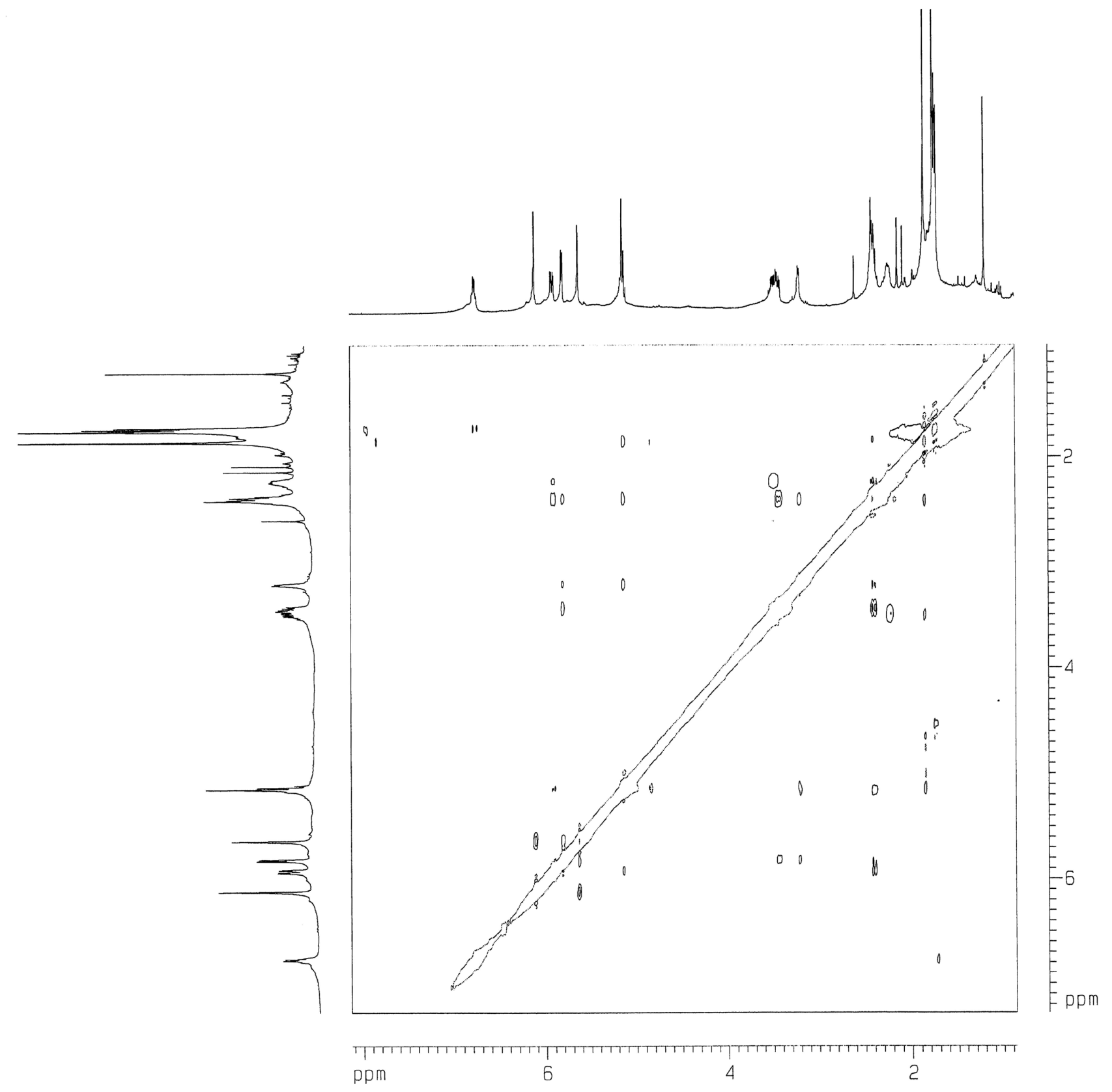

Figura 7S. Espectro de RMN NOESY $\left.\left(\mathrm{CD}_{3}\right)_{2} \mathrm{CO}\right)$ do composto 4 
MSMS: Precursor m/z --.-. /- Base Peak 359.15(1438460)

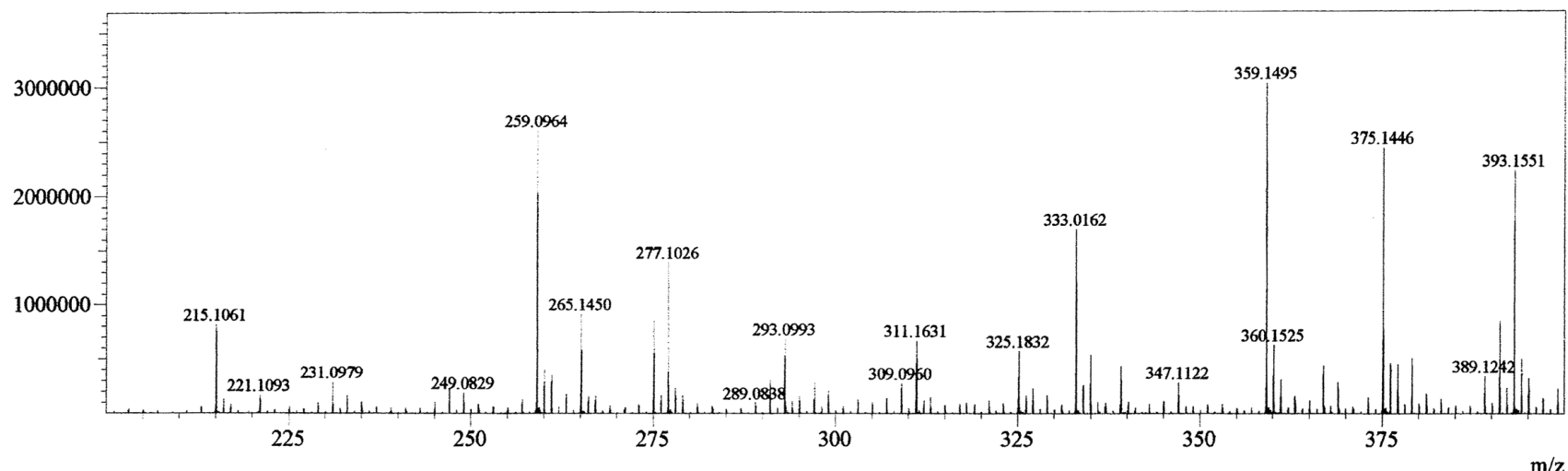

Figura 8S. Espectro de massas de alta resolução do composto 4 